\title{
Separation of cortical arteries and veins in optical neurovascular imaging
}

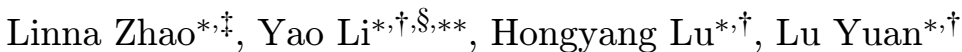 \\ and Shanbao Tong*, ${ }^{*, \boldsymbol{\oplus}, * *}$ \\ *Med-X Research Institute, Shanghai Jiao Tong University \\ 1954 Huashan Rd., Shanghai 200030, P. R. China \\ ${ }^{\dagger}$ School of Biomedical Engineering \\ Shanghai Jiao Tong University \\ 800 Dongchuan Rd., Shanghai 200240, P. R. China \\ ${ }^{\star}$ School of Life Science and Technology \\ Huazhong University of Science and Technology \\ 1037 Luoyu Rd., Wuhan 430074, P. R. China \\ §yaoli118@gmail.com \\ ฯshanbao.tong@gmail.com
}

Received 24 July 2013

Accepted 16 October 2013

Published 6 December 2013

\begin{abstract}
Separation of arteries and veins in the cerebral cortex is of significant importance in the studies of cortical hemodynamics, such as the changes of cerebral blood flow, perfusion or oxygen concentration in arteries and veins under different pathological and physiological conditions. Yet the cerebral vessel segmentation and vessel-type separation are challenging due to the complexity of cortical vessel characteristics and low spatial signal-to-noise ratio. In this work, we presented an effective full-field method to differentiate arteries and veins in cerebral cortex using dual-modal optical imaging technology including laser speckle imaging (LSI) and optical intrinsic signals (OIS) imaging. The raw contrast images were acquired by LSI and processed with enhanced laser speckle contrast analysis (eLASCA) algorithm. The vascular pattern was extracted and segmented using region growing algorithm from the eLASCA-based LSI. Meanwhile, OIS images were acquired alternatively with 630 and $870 \mathrm{~nm}$ to obtain an oxyhemoglobin concentration map over cerebral cortex. Then the separation of arteries and veins was accomplished by Otsu threshold segmentation algorithm based on the OIS information and segmentation of LSI. Finally, the segmentation and separation performances were assessed using area overlap measure $(\mathrm{AOM})$. The segmentation and separation of cerebral vessels in cortical optical imaging have
\end{abstract}

${ }^{* *}$ Corresponding authors.

This is an Open Access article published by World Scientific Publishing Company. It is distributed under the terms of the Creative Commons Attribution 3.0 (CC-BY) License. Further distribution of this work is permitted, provided the original work is properly cited. 


\section{Zhao et al.}

great potential applications in full-field cerebral hemodynamics monitoring and pathological study of cerebral vascular diseases, as well as in clinical intraoperative monitoring.

Keywords: Vessel segmentation; laser speckle imaging; optical intrinsic signals imaging; region growing algorithm; artery-vein separation.

\section{Introduction}

Cortical vessel separation is highly valuable in cerebral hemodynamics study, and plays an important role in clinical diagnostic and therapeutic management of a variety of vascular diseases, e.g., cerebral hemorrhage, stroke, aneurysm, etc. ${ }^{1}$ Therefore, an effective method is in great need to separate arteries and veins from cerebral cortical vessels.

The separation of arteries and veins has been investigated using different methods for vessel-type identification, mostly in the separation of retinal vessels. Li et al. proposed a piecewise Gaussian model for retinal vessels utilizing the central reflex characteristics to describe the image intensity distribution. ${ }^{2}$ Narasimha-Iyer et al. developed an automated methodology to identify arteries and veins from dualwavelength retinal fundus images recorded at 570 and $600 \mathrm{~nm}$ utilizing both structural and functional features of the images. ${ }^{3}$ Mendonca and Campilho proposed a set of four directional differential operators for detecting the vessel centerlines and then applied a binary morphological reconstruction method and a region growing process to segment the vessels. ${ }^{4}$

However, there has been few literature reviews on the identification of cortical vessel types based on the differences between arteries and veins in different aspects. For example, Wang et al. presented a method for separating cortical arteries and veins in optical intrinsic signals (OIS) using $0.1-\mathrm{Hz}$ oscillation at the wavelengths of 546 and $630 \mathrm{~nm}$. Multiscale matched filter and a single Gaussian model were employed to acquire different vessel types. ${ }^{5}$ Zhong et al. applied a method of independent component analysis (ICA) to OIS images and separated the arterial and venous regions. They constructed a feature vector combining heartbeat and respiration features for the fuzzy c-means clustering method and determined the vessel types using morphological intersection points. ${ }^{6} \mathrm{Hu}$ et al. identified the vesseltype based on the phenomenon that the spectral distribution of OIS was different between arterial and venous vessels. ${ }^{7}$ Vanzetta et al. identified different cortical microvascular compartments in anesthetized cats by the ratio image of two wavelengths at 540 and $560 \mathrm{~nm}$, which were corresponding to the peaks of oxyhemoglobin (HbO) and deoxyhemoglobin (HbR), respectively. Yet the absorption coefficients differences at these two wavelengths for $\mathrm{HbR}$ and $\mathrm{HbO}$ are not high enough for artery-vein separation in low signal-to-noise ratio images, e.g., rodent cerebral cortical vessels. ${ }^{8}$ Nevertheless, due to the low signalto-noise ratio, these OIS-based vessel-type separation methods have a fair performance in spatial resolution.

Compared with other imaging techniques, laser speckle imaging (LSI) provides a two-dimensional real-time, full-field cerebral blood flow (CBF) velocity map with high spatiotemporal resolution. ${ }^{9}$ The $\mathrm{CBF}$ velocity information was acquired by laser speckle contrast analysis (LASCA). While OIS imaging is a useful tool for functional brain imaging, it can obtain oxygen distribution information in vessels and tissues. ${ }^{10}$ The differences in the concentration of oxygen-hemoglobin between arteries and veins could be used to differentiate arteries and veins.

In this paper, we proposed a separation method using dual-modal optical imaging techniques, i.e., LSI and OIS, to achieve a high spatial resolution for vessel-type separation. We extracted the vessel pattern based on LSI using region growing algorithm. Then OIS images were acquired at the wavelengths of $630 \mathrm{~nm}$ and $870 \mathrm{~nm}$, so that we might distinguish the concentration of $\mathrm{HbO}$ in arteries and veins by computing the differences of reflected light intensity at $630 \mathrm{~nm}$ and at $870 \mathrm{~nm}$. Finally, arteries and veins would be separated by Otsu thresholding algorithm. Besides, we used area overlap measure (AOM) to evaluate the segmentation and separation performance.

\section{Materials and Methods}

\subsection{Animal preparation}

The experimental protocols in this study were approved by the Animal Care and Use Committee 
of Med-X Research Institute of Shanghai Jiao Tong University. One male adult Sprague-Dawaley (SD) $(240 \mathrm{~g})$ rat was used in the experiment. Firstly, the rat was anesthetized with chloral hydrate $(350 \mathrm{mg} /$ $\mathrm{kg}, \mathrm{IP}, 7 \%)$ and then mounted to a stereotaxic frame (Benchmark DeluxeTM, MyNeurolab.com, St. Louis, MO). During the experiment, the body temperature of the animal was maintained at $37 \pm 0.2^{\circ} \mathrm{C}$ using a DC control module (FHC Inc., Bowdoinham, ME, USA) and a heating pad. After removing the rat hair and epidermal tissue, the skull between bregma and lambda was polished using a dental drill (Fine Science Tools Inc., North Vancouver, Canada) and sterilized with saline until the cerebral vessels were clearly visible.

\subsection{Data collection}

The raw images were captured through the dualmodal optical imaging system (see Fig. 1), which consisted of a CCD camera (12 bit, $24 \mathrm{fps}$, exposure time, $5 \mathrm{~ms}$ ), one lens with a focal length of $8 \mathrm{~mm}$ (C240TM, Thorlabs), two light emitting diodes (LED630E, Thorlabs and LED870E, Thorlabs) powered by two driver modules (LEDD1B, Thorlabs), a laser diode (L780P010, Thorlabs) powered by a driver module (LDC220C, Thorlabs) and a computer for acquiring image data.

The cortical vessel network was focused and clearly seen by finely tuning the lens. Then the raw speckle images and OIS images at two wavelengths were alternatively captured at a sampling frequency of $24 \mathrm{fps}$ for 200 frames.

\subsection{Principles of LSI and OIS}

LSI is a real-time, full-field and high-resolution CBF imaging technology, utilizing the scattering property of the moving particles to coherent light to obtain a two-dimensional CBF velocity distribution map. ${ }^{11}$ Speckle pattern can be described using statistics since it is a random interference pattern.

According to the principle of LSI, ${ }^{12}$ speckle contrast $K$ and the $\mathrm{CBF}$ velocity obeyed the following relations

$$
K=\beta^{0.5}\left\{\frac{\tau_{c}}{T}+\frac{\tau_{c}^{2}}{2 T^{2}}\left[\exp \left(-\frac{2 T}{\tau_{c}}\right)-1\right]\right\}^{0.5},
$$

where $\beta$ represents the loss of correlation determined by the ratio of the detector size to the speckle size and polarization characteristics of scattered light, $\tau_{c}$ denotes the correlation time, $T$ is the exposure time of CCD. Bonner and Nossal ${ }^{13}$ demonstrated that the correlation time

$$
\tau_{c}=1 / a k_{0} v,
$$

where $K_{0}$ is the light wavenumber, $a$ is determined by the particle Lorentzian width and scattering properties, and $v$ is the particle velocity. When $K^{2}$ is limited to very small values, e.g., $[0,0.1],{ }^{14}$ it could be induced from Eqs. (1) and (2) that

$$
v \propto \frac{1}{K^{2}} .
$$

Equation (3) indicates that $\mathrm{CBF}$ velocity is inversely proportional to the square of speckle contrast. Therefore it is obvious that the smaller the contrast, the greater the $\mathrm{CBF}$ velocity in the two-dimensional LSI.

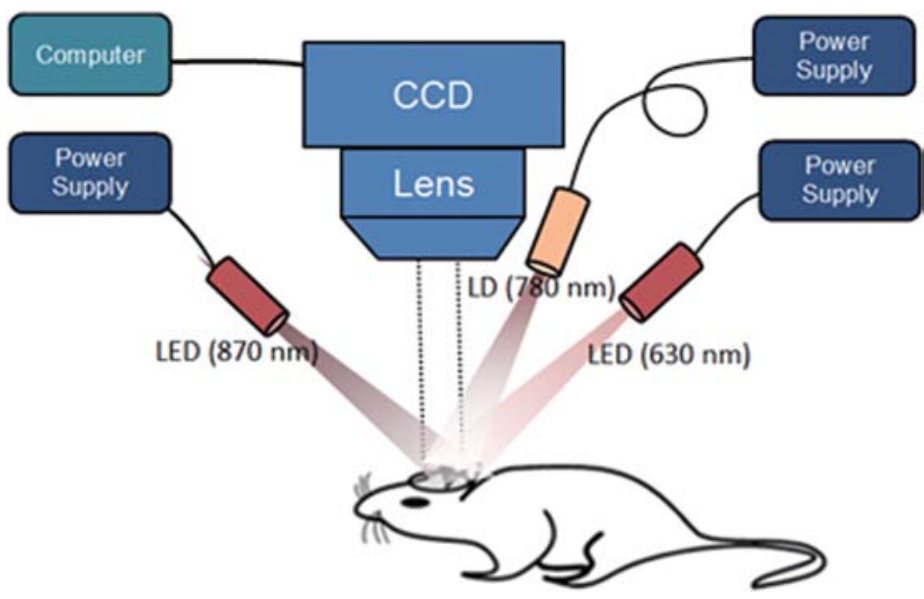

Fig. 1. Illustration of dual-modal optical imaging. 


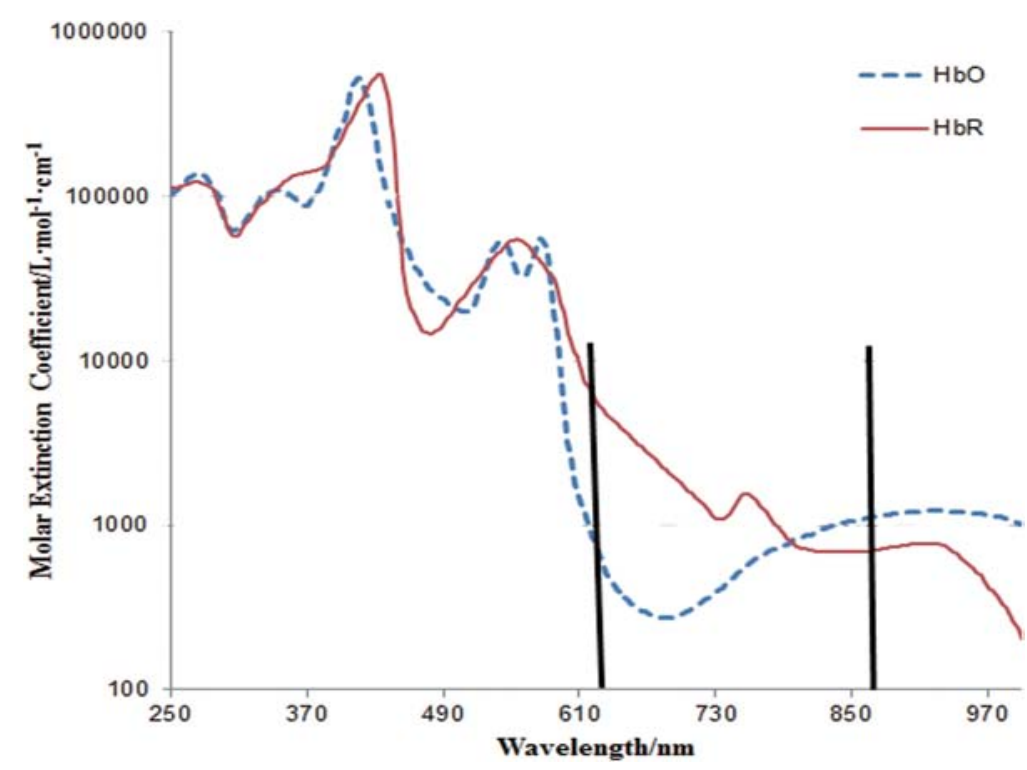

Fig. 2. The molar absorption coefficient. Data is from the website: http://omlc.ogi.edu/spectra/hemoglobin/.

Changes in neuronal activity in the cerebral cortex are coupled to changes in the blood flow and oxygenation, which provide signals that can be measured by OIS imaging. ${ }^{15}$ These intrinsic signals measured are now considered to primarily originate from variations in the concentrations of oxyhemoglobin $([\mathrm{HbO}])$ and deoxyhemoglobin $([\mathrm{HbR}]) .{ }^{16}$ Although the spatial distribution of functional activations can be qualitatively mapped with singlewavelength OIS, no quantitative information about concentration of total hemoglobin $([\mathrm{HbT}])$, oxygenation or blood flow could be obtained. To quantify relative $[\mathrm{HbO}]$ and $[\mathrm{HbR}]$, we used a method of multiple wavelengths. ${ }^{17}$
It is evident in Fig. $2^{18}$ that the absorption coefficient variation of $\mathrm{HbR}$ is much larger than that of $\mathrm{HbO}$ due to the fact that the absorption of $\mathrm{HbR}$ is more sensitive to wavelength than $\mathrm{HbO}$ within the range from 550 to $900 \mathrm{~nm}$, which can be used to detect the $[\mathrm{HbO}] /[\mathrm{HbR}]$.

\subsection{Data preprocessing}

Firstly, laser speckle contrast images were obtained by random process estimator (RPE) approach after noise reduction and registration. ${ }^{14}$ As shown in Fig. 3, the vascular network had a low gray contrast and was interfered by various noises due to

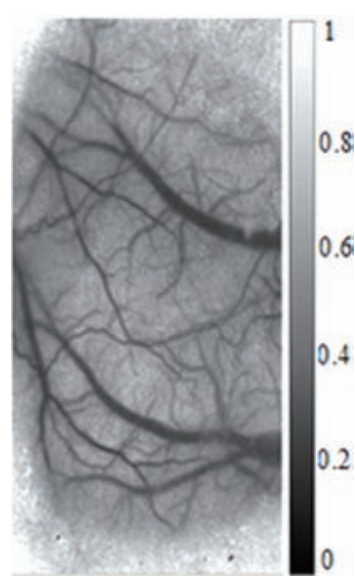

(a)

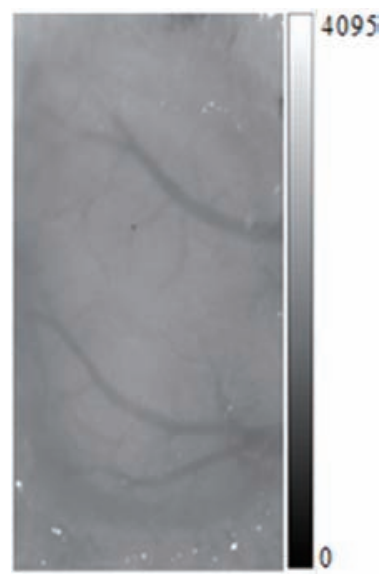

(b)

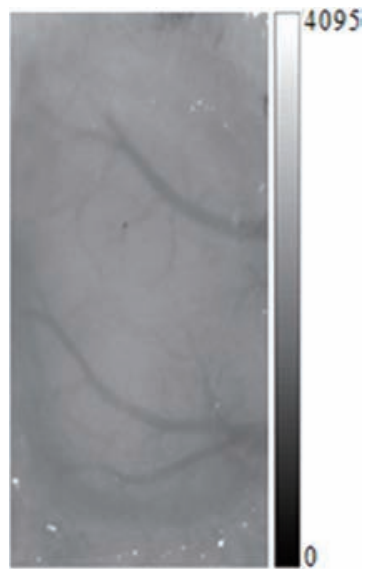

(c)

Fig. 3. LSI and OIS images (a) LSI image (b) OIS image at $630 \mathrm{~nm}$ (c) OIS image at $870 \mathrm{~nm}$. 
respiration, heartbeat and $0.1-\mathrm{Hz}$ low-frequency vessel motion. ${ }^{19}$ Hence noise reduction and image enhancement were essential to achieve good vessel segmentation performance.

\section{(1) Noise Reduction}

Noise reduction was accomplished by a linear anisotropic Gaussian filtering algorithm ${ }^{20}$ within a sliding window $(3 \times 3)$ along orientations of $0^{\circ}$, $45^{\circ}, 90^{\circ}, 135^{\circ}, 180^{\circ}, 225^{\circ}, 270^{\circ}, 315^{\circ}$, respectively. Because $0^{\circ}$ and $180^{\circ}, 45^{\circ}$ and $225^{\circ}, 90^{\circ}$ and $270^{\circ}$, $135^{\circ}$ and $315^{\circ}$ are in the same lines respectively, we only investigated gray-intensity differences along the orientations of $0^{\circ}, 45^{\circ}, 90^{\circ}$ and $135^{\circ}$ regardless of the actual vessel directions. Mean square error (MSE) along each orientation was used to determine the vessel direction

$$
\mathrm{MSE}=\sqrt{\frac{1}{N} \sum_{i=1}^{N}\left(x_{i}-\mu\right)^{2}},
$$

where $x_{i}$ represents the $i$ th pixel gray level, $N$ represents the pixels number and $\mu$ represents the mean gray level in each direction. The orientation with minimal MSE would be considered as the vessel direction. Then we implemented convolution adaptive filter with Gaussian kernel to process laser speckle contrast images. ${ }^{21}$

$$
\begin{aligned}
I(x, y)= & \int_{\infty}^{\infty} \int_{\infty}^{\infty} I\left(x^{\prime}, y^{\prime}\right) G\left(x-x^{\prime}, y\right. \\
& \left.-y^{\prime}\right) d x^{\prime} d y^{\prime},
\end{aligned}
$$

where $I\left(x^{\prime}, y^{\prime}\right)$ denotes the input image, $G$ is the Gaussian kernel and $I(x, y)$ presents the output image.

\section{(2) Image Enhancement}

The laser speckle contrast images were enhanced by utilizing enhanced laser speckle contrast analysis (eLASCA) algorithm based upon monotonic point transformation (MPT), which not only improved the CBF visualization but also reserved the CBF variability. Technical details have been reported in Ref. 22 .

The three-dimensional $K^{2}$ was reshaped into one-dimensional variable $f(i) \quad(i=1,2 \ldots M \times$ $N \times L)$ by the following equation ${ }^{22}$ :

$$
\begin{aligned}
k^{2}(m, n, l)= & f(m+(n-1) \times M+(l-1) \\
& \times M \times N),
\end{aligned}
$$

where $M \times N$ is the spatial size of an image, $L$ represents the number of total frames and $m \in$
$[1, M] ; n \in[1, N] ; l \in[1, L]$. Then Eq. (7) transformed $f$ into $f_{e}$ within a much broader range from 0 to 1 . In practice, $f_{e}$ could be approximately computed as ${ }^{22}$

$$
f_{e}=\frac{\operatorname{Num}_{f}}{M \times N \times L} \times 100 \%,
$$

where $\operatorname{Num}_{f}$ denotes the number of pixels with contrast values not more than $f$.

Finally, the enhanced laser speckle contrast images were obtained by mapping $f_{e}$ back to threedimensional $K^{2}$ according to Eq. (6).

\subsection{Vessel segmentation}

Cortical vessel segmentation was based on seeded region growing (SRG) algorithm in the following steps.

(1) Selecting Seed Points

The growing points, i.e., seed points, were manually selected by visual inspection of the images as human eyes were the gold standard to distinguish the pixels representing the vessel network. To speed up the segmentation, each image was divided into top and bottom halves so that both parts could use SRG simultaneously with three seed points in each.

\section{(2) The Homogeneity Criterion}

The pixels were merged into the growing region based on an appropriate homogeneity criterion, e.g., gray level value, region connectivity or statistical parameters. ${ }^{23}$ In this work, the homogeneity criterion was based on the vessel gray level value, and $\delta(x, y)$ was defined as the difference between merged region and its adjacent pixels, ${ }^{24}$

$$
\delta(x, y)=\left|I(x, y)-\operatorname{mean}_{\left(x^{\prime}, y^{\prime}\right) \in A}\left\{I\left(x^{\prime}, y^{\prime}\right)\right\}\right|,
$$

where $A$ is the merged region, $I\left(x^{\prime}, y^{\prime}\right)$ is the gray level of the pixel in $A, I(x, y)$ is the gray level of the adjacent point around $A$. The gray level value at the lowest trough of the image gray histogram was selected as the initial threshold. If $\delta(x, y)$ is less than the threshold, this pixel would be merged into the growing region, otherwise the pixel would not be merged. The optimal threshold was determined by estimating the degree of region overlap, i.e., AOM described in next section, between the segmented images based on algorithm and the manually segmented images. When the degree of region overlap reached the maximum, we regarded the corresponding threshold as the optimal threshold. The above process was repeated until all the pixels were tested. 
(3) The Assessment of Segmentation

It was necessary to quantitatively analyze the segmentation performance by comparing the result based on SRG with that by manual segmentation. We used AOM as the criterion for similarity measurement, ${ }^{25}$

$$
\operatorname{AOM}(A, B)=\frac{\operatorname{area}\left\{S_{A} \cap S_{B}\right\}}{\operatorname{area}\left\{S_{A} \cup S_{B}\right\}},
$$

where $S_{A}$ represents the segmentation region with SRG and $S_{B}$ represents the manual segmentation region. The manual segmentation was based on hand-sketching by a person with neurovascular observation knowledge. $\operatorname{AOM}(A, B)=1$ indicates that $S_{A}$ and $S_{B}$ are completely matching, while AOM $(A, B)=0$ means that $S_{A}$ and $S_{B}$ have no overlap.

\subsection{The separation of arteries and veins}

After the segmentation of vessels, we focused on the pixels of segmented cortical vessels by multiplying the OIS image with the well-segmented vessel binary image.

\section{(1) The Ratio of Dual-Wavelength OIS Images}

In Fig. 2, we noticed that the absorption coefficient of $\mathrm{HbR}$ was obviously higher than that of $\mathrm{HbO}$ at $630 \mathrm{~nm}$, implying that this wavelength was sensitive to the concentration change of $\mathrm{HbR}$. And the absorption coefficient of $\mathrm{HbR}$ at $870 \mathrm{~nm}$ was much lower than that of $\mathrm{HbO}$, indicating that $870-\mathrm{nm}$ light was sensitive to the concentration change of HbO. ${ }^{3,26}$

Therefore, we defined the ratio $R$ of gray level intensity at $630 \mathrm{~nm}$ to that at $870 \mathrm{~nm}$ :

$$
R=\frac{G_{630}}{G_{870}},
$$

where $G_{630}$ and $G_{870}$ represent the intensities of reflected light at 630 and $870 \mathrm{~nm}$, respectively. By applying dual-wavelength OIS imaging, we could get the distribution of relative $[\mathrm{HbO}]$ and $[\mathrm{HbR}]$ in cortical vessels for further artery-vein separation.

(2) Automatic Segmentation by Otsu Thresholding We accomplished the separation of arteries and veins using the ratio $R$ of dual-wavelength OIS images by Otsu automatic thresholding algorithm. ${ }^{27}$

Firstly, the image was divided equally into two parts in order to determine the threshold more accurately. If each OIS image $(R)$ has $N$ pixels with
$L$ gray level, the probability $p_{i}$ of each gray level could be denoted as

$$
p_{i}=\frac{f(i)}{N} \quad(i=1,2, \ldots, L),
$$

where $f(i)$ represents the number of pixels at $i$ th gray level. An initial threshold $t(1<t<L)$ was used to divide the gray levels into two groups $C_{1}$ and $C_{2}$, where $C_{1}$ contained the gray levels from 1 to $t$ and $C_{2}$ contained the gray levels from $t+1$ to $L$.

The between-class variance $\sigma^{2}$ was defined to determine the optimal threshold $t^{\prime}$ :

$$
\sigma^{2}=w_{1}\left(u_{1}-u\right)^{2}+w_{2}\left(u_{2}-u\right)^{2},
$$

where $w_{1}$ and $w_{2}$ represent total probabilities of class $C_{1}$ and class $C_{2}$, respectively, and $u$ is the mean of the whole gray levels. The threshold $t^{\prime}$ corresponded to $t$ when $\sigma^{2}$ reached the maximum value.

After the separation, AOM would be used to assess the separation performance.

\section{Results}

\subsection{Vessel segmentation using LSI}

Figure 4(c) is the segmented cortical vessel network. It is shown that major vessels were well segmented while some of the small vessels were missed. This is due to the fact that region growing algorithm is based on gray levels of the image. But from Fig. 4(a), we could hardly differentiate the small vessels from capillary bed. In our work, we selected an appropriate threshold for segmenting the major vessels.

According to Eq. (9), AOM between SRG-based separation [see Fig. 4(c)] and manual separation [see Fig. 4(b)] reached $76.59 \%$ (see Table 1), which indicated that we achieved a good segmentation performance.

\subsection{The separation of arteries and veins}

Figures 5(a) and 5(b) show the OIS vessel network at $630 \mathrm{~nm}$ and $870 \mathrm{~nm}$, respectively. We noticed that it was very difficult to distinguish arteries and veins by a single-wavelength OIS image. The ratio $R$ of OIS Images is shown in Fig. 5(c), in which the gray intensities in veins are obviously lower than those of arteries. Figure 5(d) illustrates the cortical vessel artery-vein separation using our proposed method, in which the blue vessels represent veins 


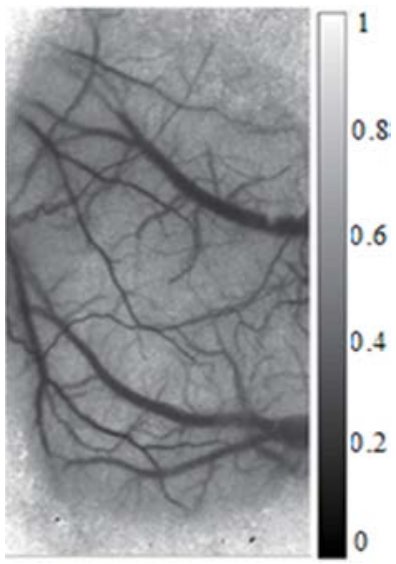

(a)

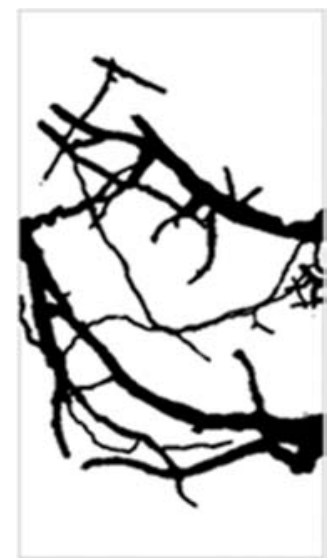

(b)

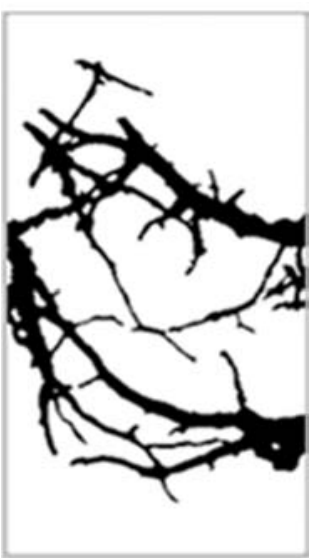

(c)

Fig. 4. Vessel segmentation (a) LSI (b) the manual segmentation (c) the segmentation with SRG.

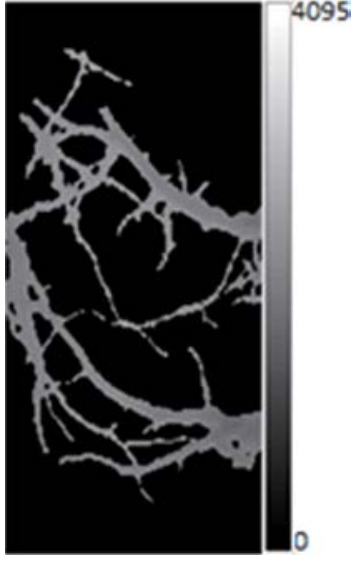

(a)

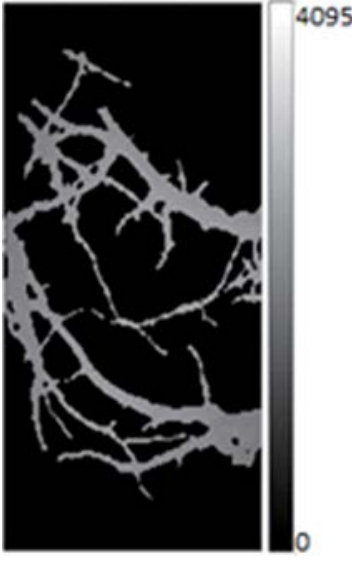

(b)

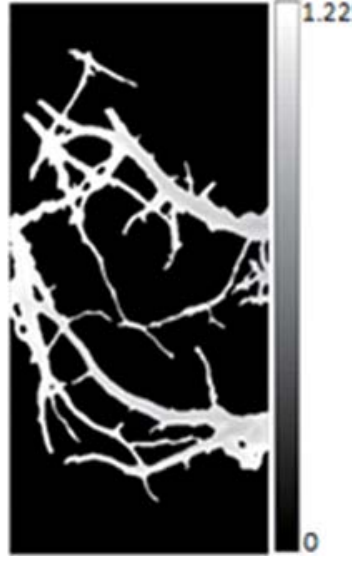

(c)

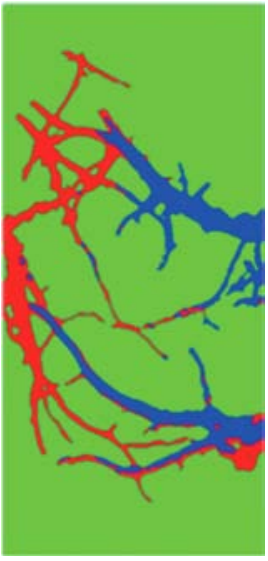

(d)

Fig. 5. The separation of arteries and veins (a) OIS vessel network at $630 \mathrm{~nm}$ (b) OIS vessel network at $870 \mathrm{~nm}$ (c) The ratio $R$ of OIS images (d) The artery-vein separation (the blue vessels represent veins and the red vessels represent arteries).

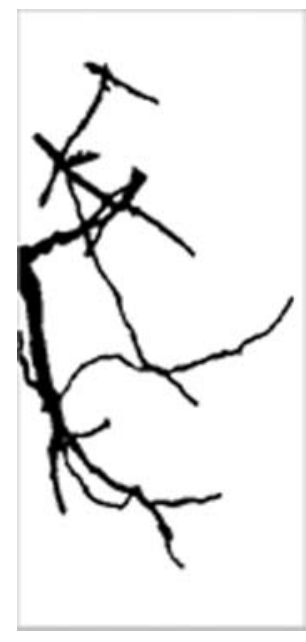

(a)

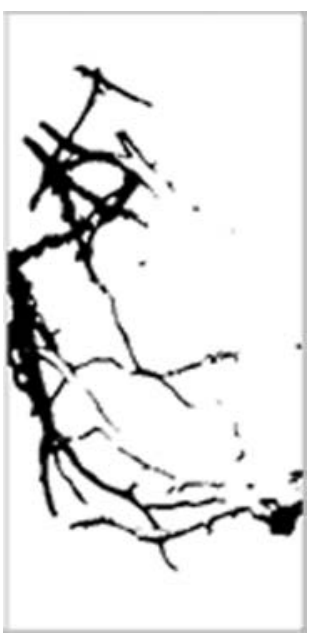

(b)

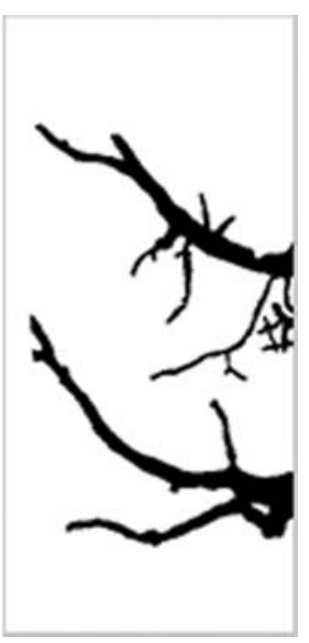

(c)

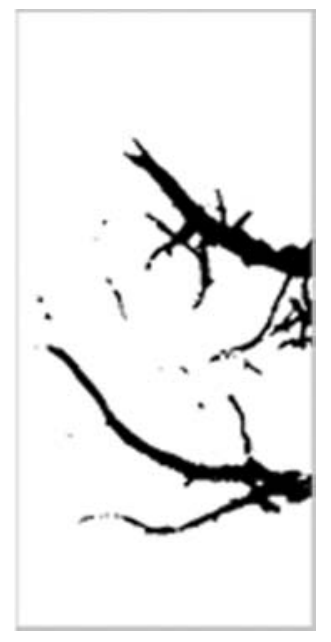

(d)

Fig. 6. Arteries and veins (a) arteries criterion (b) the segmentation of arteries (c) vein criterion (d) the segmentation of veins. 
Table 1. AOM of different vessel types.

\begin{tabular}{lccc}
\hline Vessel type & Vessels & Arteries & Veins \\
\hline AOM & $76.59 \%$ & $57.39 \%$ & $64.99 \%$ \\
\hline
\end{tabular}

Note: AOM: area overlap measure.

and the red vessels represent arteries. It was found that the major arteries and veins over the major somatosensory area were correctly separated although there were a few errors in the overlapping regions. Due to the influence of CCD depth of field, pia mater arteries and capillaries were also mistakenly classified into cortical vessels. Also, a small number of veins near the window edge were wrongly segmented as arteries due to higher noise level around the window edge area.

In Fig. 6, the arteries and veins criteria were also separated by experts according to their experiences. The overlaps of the arteries and veins were computed by Eq. (9) and the AOMs of them were $57.39 \%$ and $64.99 \%$ (see Table 1), respectively. As shown in Fig. 6(b), the segmentation of arteries was not performed optimally, among which a small part of veins were wrongly classified into arteries. By comparing Figs. 6(c) with 6(d), we found the major veins were well separated out except the edge area with low signal-to-noise ratio.

\section{Discussion and Conclusions}

In this work, we proposed an effective and efficient method for cortical artery-vein separation combining LSI with OIS imaging. Compared with other vesseltype classification methods, this method took advantage of full-field high spatiotemporal resolution LSI to segment cortical vessel network, thus achieving better performance than only OIS-based segmentation. Yet one limitation is that the SRG is a semiautomated segmentation because the seeds were selected manually. Another limitation was that the small vessels were not well segmented due to low signal-to-noise ratio between small vessels and the capillary bed while the segmentation of major vessels worked well. The classification of veins was better than arteries especially in vessel overlapping area. Because major vessels should be paid more attention during neurosurgery, our method provides a convenient and efficient way to the artery-vein separation with potential clinical and research applications.

\section{References}

1. J. F. Sabik, B. W. Lytle, P. M. McCarthy, D. M. Cosgrove, "Axillary artery: An alternative site of arterial cannulation for patients with extensive aortic and peripheral vascular disease," J. Thorac. Cardiovasc. Surg. 109, 885-891 (1995).

2. H. Li, W. Hsu, M. L. Lee, H. Wang, "A piecewise Gaussian model for profiling and differentiating retinal vessels," Proc. Int. Conf. Image Proc., Vol. 1, 1069-1072 (2003).

3. H. Narasimha-Iyer, J. M. Beach, B. Khoobehi, B. Roysam, "Automatic identification of retinal arteries and veins from dual-wavelength images using structural and functional features," IEEE Trans. Biomed. Eng. 54(8), 1427-1435 (2007).

4. A. M. Mendonça, A. Campilho, "Segmentation of retinal blood vessels by combining the detection of centerlines and morphological reconstruction," IEEE Trans. Med. Imag. 25(9), 1200-1213 (2006).

5. Y. Wang, D. Hu, Y. Liu, M. Li, "Cerebral arteryvein separation using $0.1-\mathrm{Hz}$ oscillation in dualwavelength optical imaging," IEEE Trans. Med. Imaging 30(12), 2030-2043 (2011).

6. X. Zhong, Y. Wang, M. Li, Y. Liu, "Separation of arteries and veins from optical imaging of intrinsic signals using independent component analysis," ICICIP 1, 225-229 (2011).

7. D. Hu, Y. Wang, Y. Liu, M. Li, F. Liu, "Separation of arteries and veins in the cerebral cortex using physiological oscillations by optical imaging of intrinsic signal," J. Biomed. Opt. 15(3), 036025-036025 (2010).

8. I. Vanzetta, R. Hildesheim, A. Grinvald, "Compartment-resolved imaging of activity-dependent dynamics of cortical blood volume and oximetry," J. Neurosci. 25(9), 2233-2244 (2005).

9. J. D. Briers, Webster, "Laser speckle contrast analysis (LASCA): A nonscanning, full-field technique for monitoring capillary blood flow," $J$. Biomed. Opt. 1(2), 174-179 (1996).

10. N. Prakash, F. Uhlemann, S. A. Sheth, S. Bookheimerf, N. Martinh, A. W. Togaa, "Current trends in intraoperative optical imaging for functional brain mapping and delineation of lesions of language cortex," NeuroImage 47(2), T116-T126 (2009).

11. J. D. Briers, "Laser speckle techniques in biology and medicine," Proc. SPIE 2083, 238-249 (1994).

12. D. A. Boasand, A. K. Dunn, "Laser speckle contrast imaging in biomedical optics," J. Biomed. Opt. 15 (1), 011109-011109 (2010).

13. R. Bonner, R. Nossal, "Model for laser Doppler measurements of blood flow in tissue," Opt. Appl. 20 (12), 2097-2107 (1981).

14. P. Miao, H. Lu, Q. Liu, Y. Li, S. Tong, "Laser speckle contrast imaging of cerebral blood flow in 
freely moving animals," J. Biomed. Opt. 16(9), 090502-090502 (2011).

15. A. Grinvald, E. Lieke, R. D. Frostig, C. D. Gilbert, T. N. Wiesel, "Functional architecture of cortex revealed by optical imaging of intrinsic signals," Nature 324(6095), 361-364 (1986).

16. E. M. C. Hillman, "Optical brain imaging in vivo: Techniques and applications from animal to man," J. Biomed. Opt. 12(5), 051402 (2007).

17. A. K. Dunn, A. Devor, H. Bolay, M. L. Andermann, M. A. Moskowitz, A. M. Dale, D. A. Boas, "Simultaneous imaging of total cerebral hemoglobin concentration, oxygenation, and blood flow during functional activation," Opt. Lett. 28(1), 28-30 (2003).

18. http://omlc.ogi.edu/spectra/hemoglobin/.

19. Y. Wang, D. Hu, "Separation of cortical arteries and veins using intrinsic optical signals extracted by canonical correlation analysis," ICDMA, 1082-1085 (2011).

20. J. M. Geusebroek, A. W. M. Smeulders, J. Weijer, "Fast anisotropic Gauss filtering," IEEE Trans. Imag. Process. 12(8), 938-943 (2003).

21. J. Lu, H. Lin, Z. Pan, "Adaptive region growing algorithm in medical images segmentation," J. Comput.-Aided Des. Comput. Graph. 17(10), 2168-2173 (2005).
22. P. Miao, M. Li, H. Fontenelle, A. Bezerianos, Y. Qiu, S. Tong, "Imaging the cerebral blood flow with enhanced laser speckle contrast analysis (eLASCA) by monotonic point transformation," IEEE Trans. Biomed. Eng. 56(4), 1127-1133 (2009).

23. M. Karaman, M. A. Kutay, G. Bozdagi, "An adaptive speckle suppression filter for medical ultrasonic imaging," IEEE Trans. Med. Imaging $\mathbf{1 4}$ (2), 283-292 (1995).

24. A. Mehnert, P. Jackway, "An improved seeded region growing algorithm," Pattern Recogn. Lett. 18, 1065-1071 (1997).

25. B. Sahiner, N. Petrick, H. P. Chan, L. M. Hadjiiski, C. Paramagul, M. A. Helvie, M. N. Gurcan, "Computer-aided characterization of mammographic masses: Accuracy of mass segmentation and its effects on characterization," IEEE Trans. Med. Imaging 20(12), 1275-1284 (2001).

26. J. M. Beach, K. J. Schwenzer, S. Srinivas, D. Kim, J. S. Tiedeman, "Oximetry of retinal vessels by dual-wavelength imaging: Calibration and influence of pigmentation," J. Appl. Physiol. 86, 748-758 (1999).

27. P. S. Liao, T. S. Chen, P. C. Chung, "A fast algorithm for multilevel thresholding," J. Inf. Sci. Eng. 17, 713-727 (2001). 